\title{
Approximate Constant Density Acoustic Inverse Scattering Using Dip-Dependent Scaling
}

Rami Nammour and William Symes, Rice University

\section{SUMMARY}

This abstract presents a computationally efficient method to approximate the inverse of the Hessian or normal operator arising in a linearized inverse problem for constant density acoustics model of reflection seismology. Solution of the linearized inverse problem problem involves construction of an image via prestack depth migration, then correction of the image amplitudes via application of the inverse of the normal operator. The normal operator acts by dip-dependent scaling of the amplitudes of its input vector. This property permits us to efficiently approximate the normal operator, and its inverse, from the result of its application to a single input vector, for example the image, and thereby approximately solve the linearized inverse scattering problem.

We validate the method on a $2 \mathrm{D}$ section of the Marmousi model to correct the amplitudes of the migrated image.

\section{INTRODUCTION}

The linearized inverse scattering problem assumes a known background velocity field. The background velocity field controls the kinematics of the problem: it governs the relationship between the travel times of acoustic signals and the positions of reflectors. In the case of constant density acoustics the linearized inverse problem aims at calculating the velocity perturbation. The linearized scattering operator $F$ approximately maps the true model $m$ (velocity perturbation) to the measured data $d$ (perturbation of the pressure field measured at the surface),

$$
F m \approx d .
$$

Note that $F$ depends on the background velocity field, but this dependence is suppressed for brevity. Interpreting (1) in a least squares sense yields the normal equations,

$$
F^{*} F m=F^{*} d,
$$

$F^{*}$ is a migration operator, it is adjoint to $F$. The migration operator maps the data back to the model space; $m_{m i g}=F^{*} d$ is the migrated image. $N:=F^{*} F$ is the normal operator.

Both linearized modeling and migration require the solution of a large scale PDE problems. The scale of these problems prohibits explicitly storing the normal operator as a matrix, and the use of direct matrix methods to invert it. Moreover, the expensive application of the normal operator limits the number of affordable iterations of iterative methods, as they require the application of the normal operator at each step.

The main result of this paper is a numerically efficient approximate inversion of the normal operator, based on its most important theoretical property: the normal operator preserves the discontinuities (events) in the model $m$ to which it is applied, provided that the background velocity is smooth and under some additional conditions (Beylkin, 1985; Rakesh, 1988;
Stolk, 2000). The migrated image thus contains the same events as the model and serves as a first approximation to the real model. The disagreement between the model and migrated image is due to amplitude differences. We shall show how to correct these.

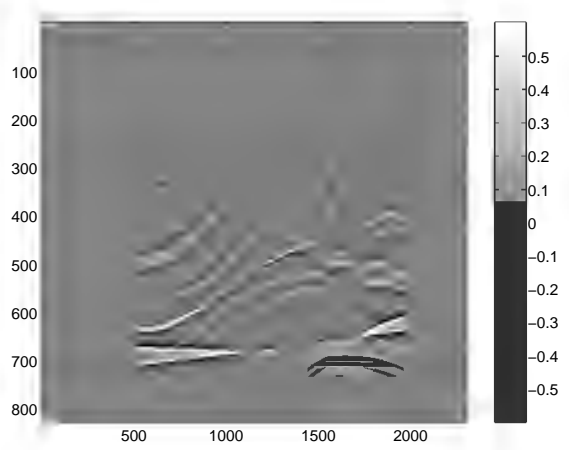

(a)

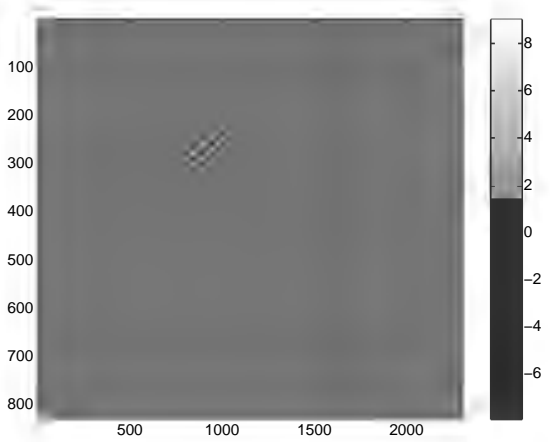

(b)

Figure 1: Comparison between the true model (a), and the migrated image (b).

\section{Example}

Figure 1 the differences between the migrated image and the true model on the Marmousi benchmark model (Versteeg and Grau, 1991). The background velocity is obtained by smoothing the velocity field, and the model (velocity perturbation) is obtained as the difference between the velocity field and its smooth part (Figure 1(a)). The model is input to a finite difference time domain Born modeling code to obtain the data. Sources and receivers positions and the recording times were similar to those used in the original synthetic model (Versteeg and Grau, 1991). The data is then migrated using a reverse time migration finite difference code, resulting in the migrated image (Figure 1(b)). Figure 1 illustrates the preservation of reflector locations between the two images. The differences between the amplitudes the of two images are also apparent. The amplitudes differ by an order of magnitude and the ampli- 


\section{Dip-Dependent Scaling}

tudes of the migrated image tend to be attenuated as a function of increasing depth.

\section{Dip-Dependent Scaling}

Previous work has established that the normal operator acts on its input vector (spatial field) by dip, frequency, and position dependent scaling (Beylkin, 1985; Rakesh, 1988; Stolk, 2000; Symes, 2008; Herrmann et al., 2008b). In view of (2) the true image differs from the migrated image by a dip and position dependent amplitude correction. The action of the normal operator on seismic images is thus approximately diagonal in phase space, the space of position, dip, and frequency. Such operators are called pseudodifferential operators. A diagonal operator can be inferred from its application to a single input vector. The choice of the migrated image as an input vector is natural as the migrated image already contains the phase space events of the true model, and we are only interested in how the normal operator scales these particular events.

We refer to the methods that estimate the inverse of the normal operator from its effect on a single input vector as scaling methods, and we refer to the approximation of the normal operator and its inverse as scaling factors. The relationship between the real model and the migrated image, is the same as the relationship between the migrated image and the remigrated image $m_{\text {remig }}=N m_{\text {mig }}$ :

$$
m=N^{\dagger} m_{\text {mig }}, \quad m_{\text {mig }}=N^{\dagger} m_{\text {remig }},
$$

where $N^{\dagger}$ is a regularized inverse of the normal operator. While the real model is not available to make use of the first relation, scaling methods use the second to estimate a scaling factor $C \approx N^{\dagger}$, and use it to approximate the solution:

$$
m_{\text {mig }} \approx C m_{\text {remig }} \Rightarrow m \approx C m_{\text {mig }} \text {. }
$$

Claerbout and Nichols (1994) and Rickett (2003) propose a scaling factor independent of dip and frequency, thus approximating the inverse of the normal operator as a multiplication by a smooth function of position.

Symes (2008) specifies the dependence on frequency as a power of the Laplacian by referring to the underlying theory (Beylkin, 1985; Stolk, 2000). The scaling method of Symes (2008) is a correction to the Claerbout and Nichols method: the normal operator is a multiplication by a smooth function only after application of a specific power of the Laplacian. However, the method suffers from one limitation: it cannot correct the amplitudes of multiple dip events, and is only successful in resolving images where dip is uniquely defined everywhere (Symes, 2008). A dip independent scaling factor acts like a dip dependent scaling factor only if the dip is unique at each point.

Guitton (2004) proposes a near diagonal approximation of the inverse of the normal operator which he calls matching filters. Matching filters amount to a phase-space scaling factor, as scaling in momentum variables is filtering. Guitton does not constrain the structure of these filters however.

Multiple dip events constitute an important class of events in seismic images; faults and point reflectors provide examples of such events. Herrmann et al. (2008b) derive a scaling method capable of resolving multiple dip events. They diagonalize the normal operator in an approximate eigen-basis, a curvelet frame. Diagonalizing the normal operator in the curvelet basis, where seismic images are sparse, renders its application efficient.

This manuscript proposes to bypass the explicit approximation of eigenvectors. The efficient application of a pseudodifferential operator is achieved by an algorithm presented by Bao and Symes (1996); we refer to this as the $\Psi D O$ algorithm. We use the $\Psi D O$ algorithm to formulate a rapidly converging optimization scheme for the scale factor. The scale factor is approximated using one application of the normal operator to the migrated image and corrects the amplitudes of the migrated image.

\section{METHOD}

Recall that the aim of this manuscript is to solve the normal equations:

$$
N m=b,
$$

where $b=F^{*} d \in \operatorname{Range}(N)$. We seek a pseudodifferential $(\Psi D O)$ scaling factor, and formulate its recovery as an optimization problem. Given the migrated image $b$ and the remigrated image $\mathrm{Nb}$,

$$
C=\underset{C \in \Psi D O}{\operatorname{argmin}}\|b-C N b\|^{2}
$$

The scaling factor $C$ is chosen from a class of pseudodifferential operators described later. In this setting the scaling factor approximates the the action of the inverse of the normal operator on the migrated image $b$. We restate this result,

$$
m=N^{\dagger} b \approx N^{\dagger} C N b \approx C N^{\dagger} N b=C b:=m_{i n v} .
$$

The first of these equations expresses the true solution $m$, the second approximate equality follows from because pseudodifferential operators approximately commute. Defining $m_{\text {inv }}:=$ $\mathrm{Cb}$ thus yields an approximation to the true model $m$. Equation (5) shows that the scaling factor approximates the action of the inverse on the normal operator on the migrated image, and it is only in that sense that $C$ approximates $N^{\dagger}$.

The optimization problem described in (4) is numerically feasible if we can apply the pseudodifferential scaling factor to the input vector $\mathrm{Nb}$ efficiently. An iterative optimization method will require at least one application of the scaling factor per iteration. The missing piece that makes the entire scheme possible is an algorithm that applies pseudodifferential operators to data efficiently.

Bao and Symes (1996) propose an algorithm for application of pseudodifferential operators efficiently in 2D. Their algorithm also extends to 3D. The class of pseudodifferential operators of interest are those defined by

$$
C u(x, z)=\iint q(x, z, \xi, \eta) \hat{u}(\xi, \eta) e^{i(x \xi+z \eta)} d \xi d \eta .
$$

Here $u(x, z)$ is the input vector (spatial field), $\hat{u}(\xi, \eta)$ is its Fourier transform. The smooth scalar function $q$ of the spatial 


\section{Dip-Dependent Scaling}

and Fourier variables, is homogeneous of degree $n$, the degree of homogeneity is the order of $C$; $q$ is known as the symbol of the pseudodifferential operator $C$. It is obvious that from (6) that pseudodifferential operators are uniquely defined by their symbol.

The $\Psi D O$ expands the symbol $q$ in the Fourier variables:

$$
q(x, z, \xi, \eta)=\sum_{l=-K / 2}^{K / 2} a_{l}(x, z) \omega^{n} e^{i l \theta}
$$

where $a_{l}$ are the first $K$ coefficients of the Fourier expansion of the symbol $q$. To derive the following expression for the approximation of the action of a pseudodifferential operator:

$$
C u(x, z) \approx \sum_{l=-K / 2}^{K / 2} a_{l}(x, z) \mathscr{F}^{-1}\left[\omega^{n-l}(\xi+i \eta)^{l} \hat{u}(\xi, \eta)\right] .
$$

Where $\omega=\sqrt{\xi^{2}+\eta^{2}}$ is the symbol of the pseudodifferential operator $\sqrt{-\nabla}$. $\mathscr{F}^{-1}$ is the inverse Fourier transform. All of these expressions are discretized in a sensible sense.

The algorithm (7) uses FFT (fast Fourier transform) and thus inherits a complexity of $O\left(K N^{2}(\log (N)+\log (K))\right)$. This complexity may be contrasted with the obvious discretization of the integral in (6) which leads to a complexity of $O\left(N^{4} \log (N)\right.$. To put things in context we may note that for the examples of interest $N \approx 10^{3}$. The number of Fourier coefficients $K$ depends on the smoothness of $q$, and is independent of $N$. The underlying theory predicts that the symbol of the normal operator is smooth and slowly varying in its arguments, and a few Fourier coefficients suffice to resolve it.

With the representation (7), the scaling factor $C$ is

- like $N^{\dagger}$ :

- pseudodifferential,

- in particular, dip dependent (scales different dips differently)

- unlike $N^{\dagger}$ :

$$
\begin{aligned}
& \text { - efficient to represent explicitly } \\
& \text { - efficient to apply to data }
\end{aligned}
$$

The order $n$ of the scaling factor is specified by the underlying theory and is the negative of to the order of the normal operator. It is interesting how the $n^{\text {th }}$ power of the square root of the Laplacian filter emerges again. In fact, keeping one Fourier coefficient, the scaling factor reduces to a power of the Laplacian filer followed by multiplication by a smooth function of position. The method thus reduces to the scaling method derived by Symes (2008) for $K=1$. The additional degrees of freedom in the Fourier coefficient of the symbol allow the method to resolve multiple dip events, capturing the dependence of the symbol on the Fourier variables.

We summarize the method as follows:

1. compute the migrated image $b$, and the remigrated image $\mathrm{Nb}$
2. represent the scaling factor by $\Psi D O$ algorithm

3. optimize for the scaling factor, $C=\underset{C \in \Psi D O}{\operatorname{argmin}}\|b-C N b\|^{2}$

4. approximate an inverse $m_{i n v}=C b \approx N^{\dagger} b=m$

See Nammour (2009) for details.

\section{EXAMPLE (CONTINUED)}

We validate the method on the $2 \mathrm{D}$ section of the synthetic Marmousi model shown in Figure 1(a). The migrated image (Figure 1(b)) shows the distorted amplitudes. Given the migrated and remigrated image, we optimize for a scaling factor to correct the amplitudes of the migrated image. We display the images on the region of interest and on the same scale, we correct the amplitudes with a scaling factor keeping one Fourier coefficient $(K=1)$ in and five Fourier coefficients $(K=5)$ in Figures 2(b) and 2(c) respectively.

It is obvious that the inverted images exhibit amplitudes in the same order of magnitude as the real model. Moreover, the amplitude is uniform as a function of depth, the events in the deeper part of the image are restored. Amplitude correction in both cases is thus successful.

The intrinsic difference between $K=1$ and $K>1$ allows the method to resolve multiple dip events for $K>1$ and not for $K=1$. The results look similar for the inverted image in both cases (Figures 2(b) and 2(c)). We therefore plot the difference between the two inverted images in Figure 3. The differences between the two images is maximal at the points of the model that exhibit multiple dip events, especially faults and intersections between two reflectors. This difference shows as either very bright spots or very dark spots. The difference of the two images picks exactly the regions of the image that exhibit multiple dips.

\section{CONCLUSIONS}

The normal operator preserves the events between the true model and the migrated image, a direct consequence of its pseudodifferential nature. It suffices to derive a scaling factor that corrects the amplitudes of the migrated image to those of the real model to achieve inversion. This manuscript describes a pseudodifferential scaling factor, a faithful representation of the inverse of the normal operator. The scaling factor thus derived is efficient to compute and to apply to data. The scaling method succeeds in correcting the amplitudes of the migrated image for a 2D section of the Marmousi synthetic model. At the places where the image exhibits multiple dip events, additional degrees of freedom in the estimated symbol allow the algorithm to correct amplitudes according to dip.

The method relies on the accuracy of the background reference velocity field, the basis for any successful linearization process to begin with. When the background velocity field is accurate, the method yields a fast and reliable approximate solution of the inverse problem. Depending on the application, 


\section{Dip-Dependent Scaling}

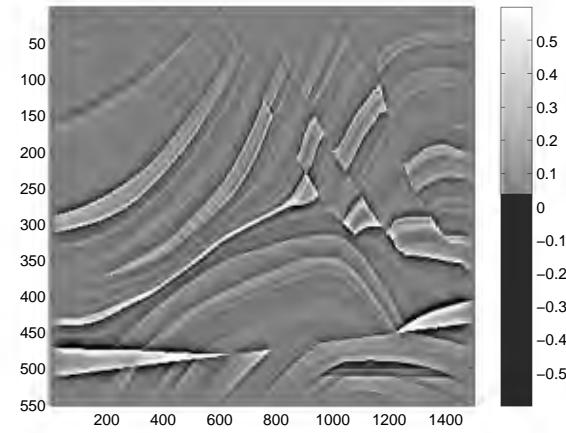

(a)

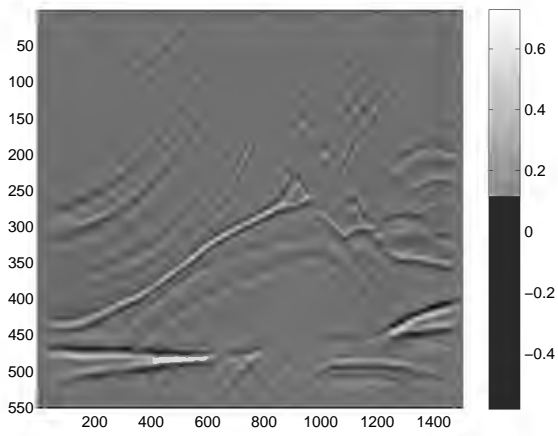

(b)

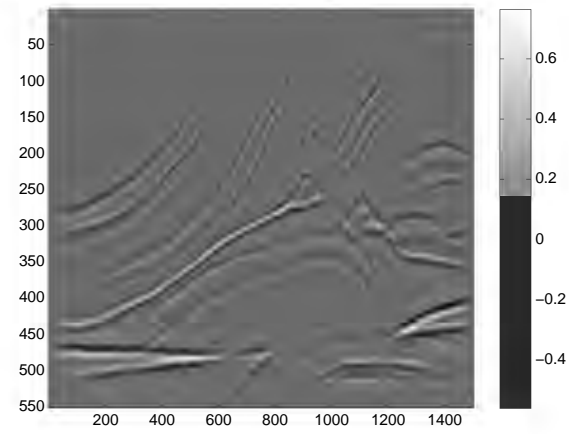

(c)

Figure 2: Comparison between the true model (a), inverse image for $K=1$ (b), and inverse image for $K=5$ (c).

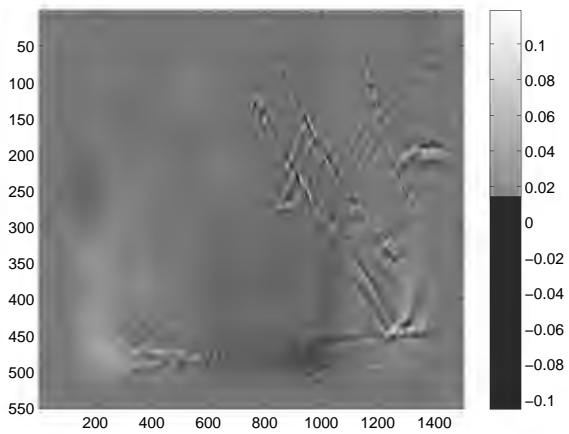

Figure 3: Difference between the inverted image for $K=1$ and $K=5$

the approximation could be used as is, or as a preconditioner to accelerate iterative refinement. Herrmann et al. (2008a) precondition a least squares linearized inverse scheme with the solution obtained with the scaling method they derive in Herrmann et al. (2008b). The approximate inverse succeeds in speeding up convergence. When the background velocity field is not accurate, the method may be used to precondition iterative methods for a Newton step in a nonlinear inversion.

The first generalization of the method deals with the 3D problem. Application of pseudodifferential operators in 3D may be achieved efficiently using spherical harmonics expansion of their symbol in analogy to the Fourier expansion in 2D. The formulation of the method translates to $3 \mathrm{D}$ without modification.

Another research direction, one we are currently involved in, deals with generalizing the method to multi-parameter recovery. One example is variable density acoustics to recover the density and compressional impedance simultaneously. With model $m$ representing a collection of two models, one for the density and one for the impedance, we seek pseudodifferential scaling factors $C_{1}$ and $C_{2}$ for which a generalization of equation (4) holds:

$$
\left\{C_{1}, C_{2}\right\}=\underset{C_{1}, C_{2} \in \Psi D O}{\operatorname{argmin}}\left\|b-C_{1} N b-C_{2} N^{2} b\right\|^{2} .
$$

Where $b, N b$, and $N^{2} b$ are given. Then,

$$
m=N^{\dagger} b \approx C_{1} b+C_{2} N b
$$

Because of the similarity of equation (8) with the definition of Krylov subspace methods in linear algebra, we term this algorithm the Operator Krylov method. This approach shows promise. 


\section{Dip-Dependent Scaling}

\section{REFERENCES}

Bao, G. and W. Symes, 1996, Computation of pseudodifferential operators: SIAM J. Sci. Comput., 17, 416-429.

Beylkin, G., 1985, Imaging of discontinuities in the inverse scattering problem by inversion of a causal generalized Radon transform: Journal of Mathematical Physics, 26, 99108.

Claerbout, J. and D. Nichols, 1994, Spectral preconditioning: Technical Report 82, Stanford Exploration Project, Stanford University, Stanford, California, USA.

Guitton, A., 2004, Amplitude and kinematic corrections of migrated images for nonunitary imaging operators: Geophysics, 69, 1017-1024.

Herrmann, F., C. Brown, Y. Erlangga, and P. Moghaddam, 2008a, Curvelet-based migration preconditioning: Technical Report 7, The University of British Columbia.

Herrmann, F., P. Moghaddam, and C. Stolk, 2008b, Sparsityand continuity-promoting seismic image recovery with curvelet frames: Applied and Computational Harmonic Analysis, 24, 150-173.

Nammour, R., 2009, Approximate inverse scattering using pseudodifferential scaling: Technical Report TR09-09, Rice University.

Rakesh, 1988, A linearized inverse problem for the wave equation: Communications on Partial Differential Equations, 13, 573-601.

Rickett, J. E., 2003, Illumination-based normalization for wave-equation depth migration: Geophysics, 68, 13711379.

Stolk, C., 2000, On the modeling and inversion of seismic data: $\mathrm{PhD}$ thesis, Universiteit Utrecht.

Symes, W. W., 2008, Approximate linearized inversion by optimal scaling of prestack depth migration: Geophysics, 73, R23-R35.

Versteeg, R. and G. Grau, 1991, Practical aspects of inversion: The Marmousi experience: Proceedings of the EAEG, The Hague. 